\title{
Sliver Solar Cells
}

\author{
Evan Franklin, ph 61261254883 evan.franklin@anu.edu.au \\ Andrew Blakers, ph 61261255905 andrew.blakers@anu.edu.au \\ Vernie Everett, ph 61261258872 vernie.everett@anu.edu.au \\ Klaus Weber, ph 61261259734 klaus.weber@anu.edu.au \\ Centre for Sustainable Energy Systems, Australian National University \\ Canberra, 0200, Australia. Fax 61261258873
}

\begin{abstract}
Sliver solar cells are thin, mono-crystalline silicon solar cells, fabricated using micro-machining techniques combined with standard solar cell fabrication technology. Sliver solar modules can be efficient, low cost, bifacial, transparent, flexible, shadow-tolerant, and lightweight. Sliver modules require only 5 to $10 \%$ of the pure silicon and less than $5 \%$ of the wafer starts per $\mathrm{MW}_{\mathrm{p}}$ of factory output when compared with conventional photovoltaic modules. At ANU, we have produced 20\% efficient Sliver solar cells using a robust, optimised cell fabrication process described in this paper. We have devised a rapid, reliable and simple method for extracting Sliver cells from a Sliver wafer, and methods for assembling modularised Sliver cell sub-modules. The method for forming these Sliver sub-modules, along with a lowcost method for rapidly forming reliable electrical interconnections, are presented. Using the sub-module approach, we describe low-cost methods for assembling and encapsulating Sliver cells into a range of module designs.
\end{abstract}

Keywords: Solar, Photovoltaic, Sliver Cell, Silicon, Thin-film

\section{INTRODUCTION}

For decades crystalline silicon has been the material of choice for photovoltaic (PV) modules, and currently accounts for $90 \%$ of the PV market. Silicon-based solar cells are popular because of high cell efficiencies compared with alternative materials, proven long-term cell and module reliability, the abundant and non-toxic nature of silicon, and the ability of the silicon-based PV industry to share technology, skilled people, and infrastructure with the silicon-based integrated circuit industry.

In order for PV technology to compete economically with conventional sources of electricity, one of two scenarios must be established: either conventional electricity must be supplied at its true cost - including the cost of environmental damage, or the cost of solar module production must be reduced to around US\$1 per watt. Given that approximately half the cost of today's solar module is attributable to the hyper-pure silicon required to produce it ${ }^{1}$, a common driver for considerable current PV research is to realise a significant reduction in the consumption of silicon for a given module size. A promising option for achieving this goal is thin crystalline silicon solar cells. Much effort expended worldwide towards large reductions in the silicon content of PV modules has been directed towards thin-film silicon deposition and ribbon silicon ${ }^{2-4}$. Sliver technology, developed at the Centre for Sustainable Energy Systems at the Australian National University (ANU) ${ }^{5-7}$, offers an alternative method for vast reductions in silicon consumption via a 10 - to 20 -fold increase in the utilisation of conventional crystalline silicon.

\subsection{Sliver technology}

Sliver cells are long, narrow, thin mono-crystalline silicon solar cells capable of high ( $>20 \%)$ efficiencies. Sliver cells are fabricated from mono-crystalline wafers in a rather different manner to conventional wafer-based solar cells, but nevertheless utilising standard fabrication techniques. Rather than fabricating a single solar cell on the surface of a wafer, many hundreds - up to several thousand - of individual Sliver solar cells are fabricated within a single wafer. The dimensions of a Sliver cell depend upon wafer size, wafer thickness and the Sliver substrate formation or patterning method used. Sliver cells typically have a length of $5-12 \mathrm{~cm}$, a width of $0.5-2 \mathrm{~mm}$, and a thickness of $20-100 \mu \mathrm{m}$. These very thin Sliver cells are symmetrical, perfectly bi-facial, have edge-positioned non-shading electrodes and a large surface area to mass ratio.

Device and Process Technologies for Microelectronics, MEMS, Photonics, and Nanotechnology IV edited by Hark Hoe Tan, Jung-Chih Chiao, Lorenzo Faraone, Chennupati Jagadish, Jim Williams, Alan R. Wilson Proc. of SPIE Vol. 6800, 680010, (2008) · 0277-786X/08/\$18 - doi: 10.1117/12.759594 
Figure 1 is a depiction of a wafer with, for the sake of ease of discussion, just a few Sliver cells represented. The essential step in forming the Sliver substrates is to form deep narrow grooves all the way through the wafer.

A variety of techniques can be used including, but not limited to, narrow focus laser cutting, water-jet guided-beam laser cutting, narrow blade dicing saw, anisotropic alkaline etching, or high-speed plasma etching. Several of these methods, separately and in combination, have been used at ANU to reliably create multiple narrow grooves less than $50 \mu \mathrm{m}$ wide through $1 \mathrm{~mm}$ thick wafers on a pitch of $100 \mu \mathrm{m}$, leaving Sliver substrates approximately $50 \mu \mathrm{m}$ thick and $1 \mathrm{~mm}$ wide secured at their ends by the remaining wafer frame.

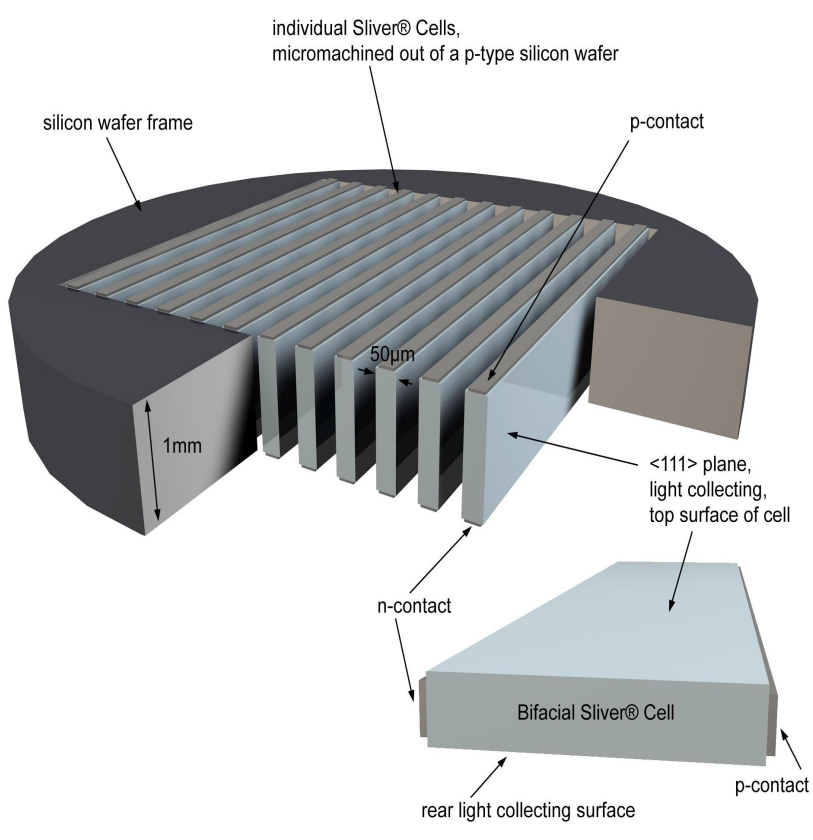

Figure 1. A wafer containing Sliver cells. The pn junction is located beneath each large, light collecting surface.
Individual Sliver solar cells are created from the narrow strips of silicon formed during the grooving process, with most processing steps completed while the strips are still supported by the wafer frame. All cell processing steps are based on standard silicon solar cell processing technologies. Solar cell electrodes are also formed while the Sliver cells are retained in the wafer, with the main surfaces of the wafer being metallised to form contacts along the long narrow edges of the cells. Following extraction from the wafer, the Sliver cell is rotated about its long axis. The large face of the Sliver cell, corresponding to the sidewall formed by grooving, becomes the sun-facing surface of the cell. The combined surface area of the Sliver cells created within the wafer can therefore be many times greater than the original wafer surface area. Since the processing treatment of both sidewalls of each Sliver cell is identical, the cell is, by default, perfectly bifacial.

Because the Sliver cell is very thin and has pn-junctions on both large faces, corresponding to the sidewalls of each groove, good surface passivation ensures that internal quantum efficiency is essentially unity across the spectrum. In contrast to conventional solar cells, with the exception of rear-contact solar cells, there is virtually no

shading of the cell due to metallisation since the metal contacts, only 1-2 $\mu \mathrm{m}$ thick, are on the long narrow edges of the Sliver cell rather than the wide sunward facing surface. The edges of each cell occupy only a very small fraction of the total surface of the cells, and doping can be very heavy below the metal contacts. Excellent, low-resistivity contacts and minimal recombination are thus readily achieved. Good short-circuit currents, high open-circuit voltages, and high Sliver cell efficiencies are observed as a result.

Recently the authors became aware of a 1981 paper by Iles \& Soclof ${ }^{8}$ that describes a general concept for area multiplication of a silicon wafer, and also describes a number of processes for achieving an increase in wafer surface area. The processes described by Iles \& Soclof, whilst possessing benefits for silicon utilisation, also recognise a number of significant problems including highly non-uniform etching of the grooves, difficulty in holding the thin strips of silicon in place during wafer processing, an inability to texture the hidden (sunward) faces in the grooves, and difficulty in fabricating solar cells on the thin strips.

Blakers and Weber $^{9}$ at the Australian National University (ANU), financially supported by the Australian company Origin Energy and the Australian Research Council, independently developed the concepts described by Iles \& Soclof and have substantially improved upon this earlier work, including solving the above-listed problems.

\subsection{Sliver cell application}

The unusual shape of Sliver cells means that they can be used to produce novel module designs. One such Sliver module design utilises a very simple rear-surface Lambertian reflector, with Sliver cells occupying only a fraction (typically half) of the module surface area $^{10}$, as depicted in the diagram of Figure 2. This module design allows for a further reduction in silicon usage: the number of Sliver cells required can be halved, while high optical efficiency is retained via the rear- 
surface, diffuse-reflection and light-trapping regime. The Lambertian-reflector module efficiency will depend not only on the total reflectivity of the rear surface, but the diffuse - or Lambertian - reflectivity properties, as well as cell spacing and cell efficiency ${ }^{10}$.

An alternative module design comprises a similar arrangement of Sliver cells within the module, but features a transparent rear glass sheet rather than a Lambertian reflective surface. This design produces a semi-transparent module, with considerable architectural potential. The degree of transparency can be simply "dialled-up" by varying the spacing between individual Sliver cells. Yet another module design utilises thin, flexible plastic sheeting to encapsulate Sliver cells, thereby creating flexible, rollable, or wearable solar modules. An example of a small flexible module is provided in Figure 3.

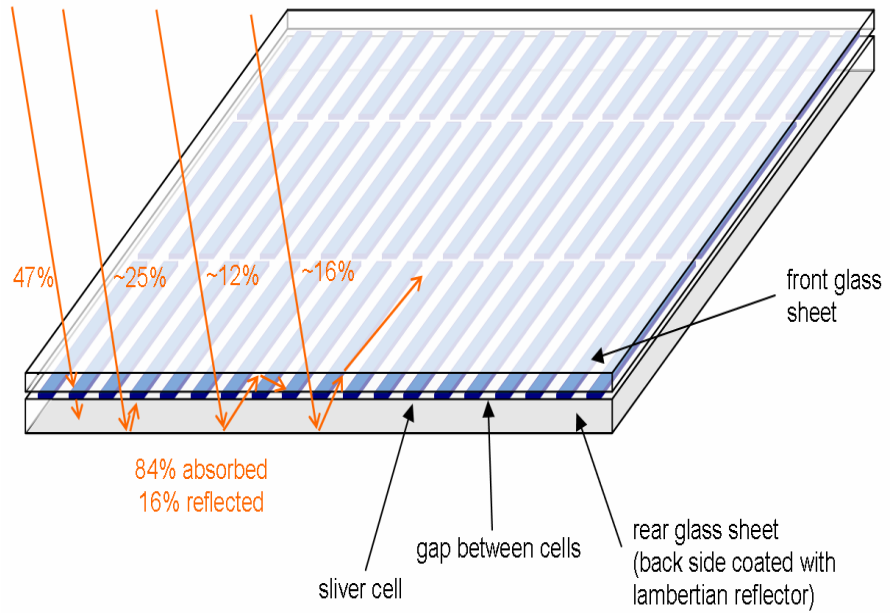

Figure 2. A Sliver cell module with $50 \%$ cell coverage, showing modelled absorption and reflection fractions for possible light pathways ${ }^{10}$.

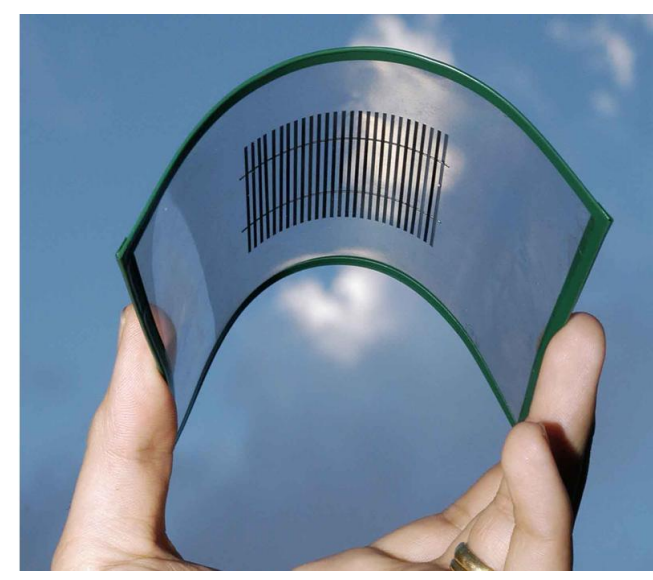

Figure 3. An example of a flexible, submodule based Sliver cell module.

\subsection{Advantages of Sliver technology}

Sliver technology allows for the possibility for better than a tenfold reduction in silicon usage, and a 20 - to 40 -fold decrease in the number of wafers processed per MW, compared with standard crystalline-silicon wafer-based PV technology. Additionally, far fewer Sliver wafers need to be processed in order to obtain the same active solar cell surface area as that produced by conventional wafer processing methods. This significantly reduces cell processing cost per unit area of cell produced. Lastly, Sliver cells are highly efficient and therefore capable of producing more electrical power for a given cell area or module area when compared with conventional solar cells.

Part of the reductions in material and manufacturing costs are achieved by innovative module designs made possible by the unusual size, shape, and operating characteristics of Sliver cells. For example, a module with a rear-surface Lambertian reflector and evenly spaced Sliver cells can capture over $80 \%$ of incident light with only $50 \%$ of the module area being covered by cells ${ }^{10}$.

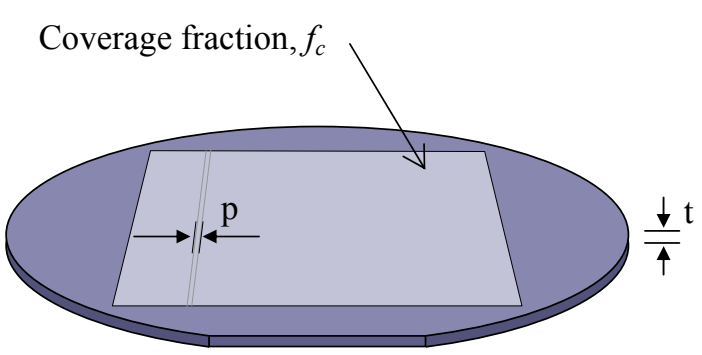

Figure 4. Key wafer parameters for determining area multiplication
The approximate area multiplication factor $A_{\text {mult }}$ for the Sliver process is given by the expression $A_{\text {mult }}=f_{C} \cdot \frac{t}{p} \cdot \frac{1}{C}$

Here $f_{c}$ is the proportion of the wafer that is utilised, $t$ is the wafer thickness and $p$ is the groove pitch (the centre to centre spacing) as shown in Figure 4. The proportion of a module covered by Sliver cells is denoted by $C$. With mature Sliver technology the values of these parameters could be $80 \%, 1500$ microns, 40 microns and 50\% respectively, yielding an area 
multiplication factor $A_{m u l t}$ of 60 . A single $15 \mathrm{~cm}$ diameter wafer would contain enough Sliver cells to populate an entire $1 \mathrm{~m}^{2}$ module, compared with 60 wafers processed using conventional technology.

Such large reductions in silicon usage and wafers processed per $\mathrm{MW}_{\mathrm{p}}$ capacity easily justifies the extra expense associated with the use of moderate- to high-quality silicon, and the application of wafer processing methods directed towards optimising the fabrication of high-efficiency solar cells. The result of this integrated approach is that high cell efficiencies can be obtained at a significantly reduced $\$ / \mathrm{W}_{\mathrm{p}}$ process cost.

\section{SLIVER CELL FABRICATION}

A successful Sliver cell manufacturing process must be robust and reliable, with maximum width process windows, a high process yield, and a high and uniform cell performance. Further, the reliance on non-standard solar cell manufacturing techniques and non-standard equipment should be minimised in order to capitalise on existing knowledge and understanding, and to minimise the introduction of uncertainties. Successful transfer of Sliver technology to an industrial manufacturing environment hinges on meeting these two broad requirements.

\subsection{Details of the fabrication process}

A robust, optimised Sliver cell processing sequence has been developed at ANU. This is comparable in complexity to other high-efficiency solar cell fabrication processes. A summary of the stream-lined Sliver cell processing sequence is listed in Table 1. Following etching to remove wire-saw damage, the wafers are exposed to a phosphorus diffusion designed to produce a heavily doped region below eventual n-contacts. A final sheet resistance of less than $15 \Omega / \square$ is a typical target for this diffusion. The diffused layer of silicon is then removed from one wafer surface only. Boron is then diffused into the exposed surface for eventual p-contacts. For this diffusion, a sheet resistance of around $40 \Omega / \square$ is a typical target. Following these preparation steps, a key Sliver process step, where grooves are etched all the way through the wafer, is performed. The grooving process is implemented by first creating either laser-defined or photolithographydefined openings in a dielectric mask, which can be either oxide or nitride. This mask can be either grown or deposited on both wafer surfaces. Formation of the Sliver grooves is performed by etching the grooves in a highly anisotropic hydroxide etch solution. At this point the sidewalls of the newly formed Slivers ideally require texturing. Although standard (100) texturing techniques cannot be applied to anisotropic-etched Sliver sidewalls (which have $<111>$ orientation), excellent texturing and light-trapping ${ }^{11}$ can instead be achieved via an acid etch technique through a very thin deposited silicon nitride layer ${ }^{12}$. Alternative simplified methods are presently under development at ANU.

\begin{tabular}{cl}
\hline Step & Step description \\
\hline $\mathbf{1}$ & Wafer etch and clean \\
$\mathbf{2}$ & Heavy phosphorus diffusion and in-situ oxide growth \\
$\mathbf{3}$ & Selective removal of diffusion from one wafer surface \\
$\mathbf{4}$ & Heavy boron diffusion \\
$\mathbf{5}$ & Groove mask formation \\
$\mathbf{6}$ & Initiate grooves \\
$\mathbf{7}$ & Groove formation \\
$\mathbf{8}$ & Sidewall texture \\
$\mathbf{9}$ & Sidewall emitter phosphorus diffusion \\
$\mathbf{1 0}$ & ARC growth / deposition \\
$\mathbf{1 1}$ & Remove dielectric from both wafer surfaces \\
$\mathbf{1 2}$ & Metallise wafer surfaces (the Sliver ${ }^{\circledR}$ cell edges) \\
\hline
\end{tabular}

Table 1. An optimised processing sequence overview

The pn junctions on both Sliver sidewall surfaces are formed via a light phosphorus diffusion, which can be tailored to suit a given application. This diffusion is driven in to a depth of around $1 \mu \mathrm{m}$. An anti-reflection coating is formed on the Sliver sidewalls while the Sliver substrates are still retained within the wafer frame. PECVD nitride is a non-conformal deposition; that is, it will not coat all surfaces uniformly regardless of the surface topology. Therefore, in applying a 
nitride AR coat to Sliver cells, it is necessary to use LPCVD SiN deposition. However, LPCVD SiN is notorious for producing poor surface passivation. In order to counter the poor $\mathrm{SiN}$ surface passivation, a thin $\mathrm{SiO}_{2}$ layer is grown during the emitter drive-in step, immediately prior to SiN deposition, producing a very effective oxide/nitride stack ${ }^{13-16}$.

In preparation for metal contacts to be formed on the top and bottom wafer surfaces, the dielectric layer must first be removed. Reactive Ion Etching (RIE) is a well-known, highly directional etch method. RIE can be used to remove top and bottom $\mathrm{SiN}$ and $\mathrm{SiO}_{2}$ layers without affecting the Sliver sidewall ARC. Finally, metallisation is achieved by the evaporation of contact metals onto both top and bottom surfaces of the prepared wafer. Any metal which lands on Sliver sidewalls can removed using a selective metal etch solution. Alternatively, the metal evaporation can be conducted at an angle to the wafer surface so that any of the metal depositing on the Sliver faces only extends part-way down one Sliver sidewall from each evaporation direction, thus preventing shading on what will eventually become the front surface of each cell.

During the development of the present iteration of the cell fabrication process, several key process issues were identified which were directly related to specific intricacies of the cell design and cell fabrication processes. For high efficiency solar cells, emitter doping should be light enough to ensure good surface passivation and high transparency for photogenerated minority carriers. However, for such wide contact spacing, a lightly-doped emitter can result in significant series resistance losses, which manifest themselves as distributed series resistance ${ }^{17}$, reducing fill-factor and cell efficiency accordingly. This is a particular loss mechanism for Sliver cells greater than about $1 \mathrm{~mm}$ wide, or for Sliver cells operating under concentrated illumination where the higher currents see elevated effective resistance. The resistance loss is proportional to illumination intensity and to the square of the Sliver width. Even at 1-Sun intensity with $1 \mathrm{~mm}$ wide cells, the series resistance of the emitter regions alone can easily account for some 3 or 4 fill-factor points - or even higher - for poorly-controlled, or light, emitter diffusions.

Owing to the unique topology of Sliver-patterned wafers, Sliver cell processing can lead to higher than expected emitter losses. Measurements have shown that the level of emitter doping of Sliver sidewalls, which are diffused while the Sliver substrates are still held in the wafer frame, is considerably lower than that for normal wafer surfaces in the same process. Values of the sheet resistance, measured on an actual Sliver sidewall using a carefully calibrated four point probe process developed for this purpose, are, as a rule, observed to be about twice that of the sheet resistance measured on a planar wafer exposed to the same diffusion.

Another significant loss mechanism identified in Sliver cells is significant non-ideal recombination located in a region of heavily-compensated silicon, which arises due to the overlap between the phosphorus diffusion on the groove sidewalls and the boron diffusion at the Sliver cell edge. Without careful control and optimisation, this overlap region can result in cells with reduced fill-factor, characterised by a distinct high $\mathrm{n}$-factor recombination component on a measured $\mathrm{J}_{\mathrm{SC}}-\mathrm{V}_{\mathrm{OC}}$ curve. Careful monitoring and control of both of these diffusions is required to ensure that associated recombination losses are minimised.

\subsection{Sliver Cell performance}

Sliver cells with high open-circuit voltage and high fill-factor have been consistently produced. Equally importantly, the processing sequence has been shown to deliver a high process yield, with excellent consistency in the measured performance between Sliver cells within a given wafer, and between wafers across different batches. While no complete statistical analysis has been conducted as yet, testing between 10 and 20 Sliver cells per wafer reveals cell performance variance within a few percent. A high yield and uniform performance consistency, such as that demonstrated by the cell fabrication process described here, is crucial for realising a cheap, high through-put, robust, reliable, and simple Sliver cell handling process, electrical interconnection and module design and construction method that is well-suited to an industrial environment.

We have recently fabricated Sliver cells with efficiencies exceeding $20 \%$. These cells were made from $1 \mathrm{~mm}$ thick wafers, and incorporated an LPCVD SiN $(\mathrm{n} \approx 2.1)$ anti-reflection coating. The cells were fabricated without surface texturing, but included the application of a post-process, rear-surface, Lambertian reflection coating. A highly reflective, high-performance, white Lambertian coating is easily applied to one face of the Sliver cells once they have been removed from the wafer. While not as effective, in terms of light-trapping characteristics, as excellent surface texturing, the diffuse reflective Lambertian coating does produce a very good short-circuit current in Sliver cells. Median and standard deviation data for six cells produced using our present optimised processing sequence are presented in Table 2, 
while the corresponding IV curves are plotted above in Figure 5. Calibrated in-house cell measurements only are reported.

We believe there is a clear path to $22 \%$ laboratory cell efficiencies. Production cell efficiencies of $21 \%$ are clearly possible in a manufacturing environment, given the care that can be afforded even in a production environment because of the large effective cell surface area contained in each wafer. In terms of cost per unit power output, the high and uniform cell efficiencies attainable with the process described here provide a significant extra advantage beyond the reduced silicon consumption which is the key feature of Sliver technology.

\begin{tabular}{lllll}
\hline $\begin{array}{l}\text { Median } \\
\text { values }\end{array}$ & $\begin{array}{l}\text { Voc } \\
(\mathrm{mV})\end{array}$ & $\begin{array}{l}\text { Jsc } \\
(\mathrm{mA} / \mathrm{cm} 2)\end{array}$ & FF & Eff \\
\hline @ 1 sun & 666 & 37.0 & 0.82 & $\begin{array}{l}20.1 \% \\
\text { Std Dev }\end{array}$ \\
\hline
\end{tabular}

Table 2. Measured data for six Sliver cells fabricated using the simplified processing sequence.

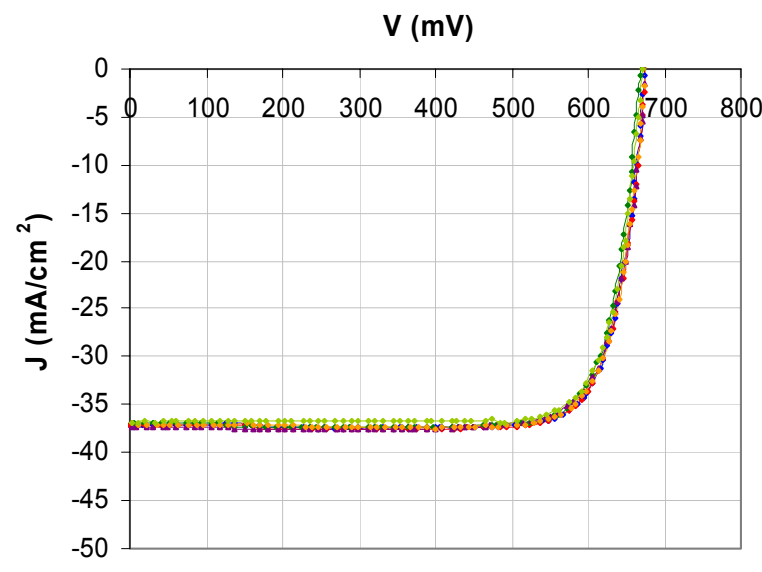

Figure 5. Measured IV curves for six Sliver cells fabricated using optimised processing sequence.

\section{SLIVER MODULE CONSTRUCTION}

Consideration of optimisation strategies for cell fabrication and module production processes cannot be made independently or in isolation, since the most cost-effective module production methods also rely upon a cell fabrication sequence which deliver high-efficiency cells with high yield and uniform, or low-variance, cell performance spreads. A high yield minimises per unit cost, and a consistent performance in turn avoids the necessity of measuring and binning every cell.

The existence of a fast, reliable and inexpensive construction method for producing Sliver modules is an essential ingredient in the commercial success of a Sliver cell fabrication facility. Industrial solar module manufacturers have traditionally relied heavily upon the manual testing, binning, laying-out, tabbing and interconnection of cells, although some large manufacturers have now incorporated automated testing and binning into their production facilities. For Sliver cell module assembly, manual handling is simply not an option: the sheer number of Sliver cells precludes the use of manual handling in any realistic commercial environment, so some degree of automation is required.

A Sliver solar power module constructed from Sliver cells will contain between 5,000 and 10,000 cells per square metre of module area. Compared with 60 to 80 conventional solar cells for the same module area, it is immediately obvious that a radically different assembly process is essential. Furthermore, because of the physical properties of the Sliver cells, conventional cell-handling methods and PV module designs cannot be used. As an indication of the scale of the task of handling Sliver cells, a $100 \mathrm{MW}$ capacity manufacturing plant would need to process in the order of 150 Sliver cells per second. It is a significant engineering challenge to devise a method for separating, testing, binning, assembling, and electrically interconnecting this very large number of solar cells in a rapid, reliable, and cost-effective manner. A simplified modular approach to Sliver cell separation, handling, assembly, and electrical interconnection has been developed $^{18,19}$.

\subsection{The Sliver sub-module concept}

Methods for separating, handling, assembling, and electrically interconnecting large numbers of Sliver cells in a lowcost, efficient, robust, and reliable manner have been developed. Rather than separating individual Sliver cells from the wafer using an expensive, high-speed, automated process such as the process described above, modular sub-assemblies are constructed, which can be thought of as conventional solar cell analogues.

The sub-module approach provides a means for the low-cost separation, handling, assembly, and electrical interconnection of groups of Sliver cells to form a conventional solar cell analogue that, because of its appearance, is called a "Raft"18. Origin Energy has subsequently also published a sub-module approach ${ }^{20}$. 
The Raft modular sub-assemblies comprise essentially planar arrays of parallel Sliver cells oriented orthogonal to, and affixed on, a supporting medium. These planar arrays are comparable in size to a conventional solar cell. The supporting medium can be a collection of long, thin material in the form of a ribbon or a track, or it may be quite wide, up to slightly larger than the size and shape of the Sliver cell array.

Extraction of the Sliver cells from the host wafer can be accomplished at very low cost using a variety of techniques ${ }^{18}$. The key requirement for any simplified handling concept requires maintaining each Sliver cell in a well-defined position relative to its neighbours, and entirely avoiding the requirement for handling individual cells. One method is described below, by way of example.

Adhesive tape can be placed on one or both surfaces of the host wafer to retain the relative location of adjacent cells. The tape engages the edge of each Sliver cell. The ungrooved periphery of the wafer is then removed using a laser, dicing saw or simple mechanical fracture. The one or two pieces of adhesive tape hold each Sliver cell firmly in position relative to adjacent Sliver cells by attachment to each edge, corresponding to the faces of the wafer, as shown diagrammatically in Figure 6. An image of an actual transfer process is shown in Figure 7.

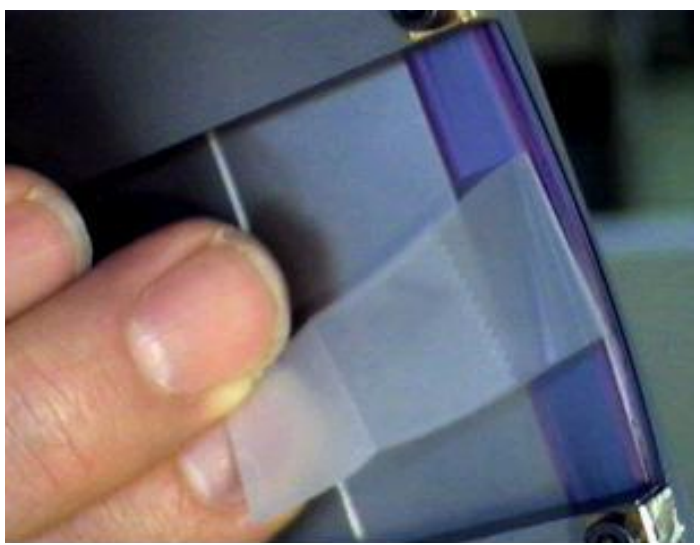

Figure 7. Adhesive tape transfer of Sliver cells and loading a single-stack cassette.

The Rafts can be readily formed using an array of singlestack cassettes or, alternatively, a multi-stack cassette ${ }^{18}$, a prototype version of which is shown in Figure 9. For the example where an array of single-stack cassettes is used, 50 cassettes full of $100 \mathrm{~mm}$ long Sliver cells are placed side by side so that the top (or bottom) Sliver cells form a planar array of Sliver cells with the required spacing, say on a $2 \mathrm{~mm}$ pitch, so that $100 \times 100 \mathrm{~mm}$ Rafts are formed. A pair of pre-metallised silicon crossbeams, $104 \mathrm{~mm}$ long, is adhered to the top (or bottom) Sliver cell of each cassette and the secured planar array is then withdrawn, with the 50 Sliver cells arranged and secured in a Raft format. A lowcost solder process has been devised that can be used to electrically interconnect each of the 50 Sliver cells to the

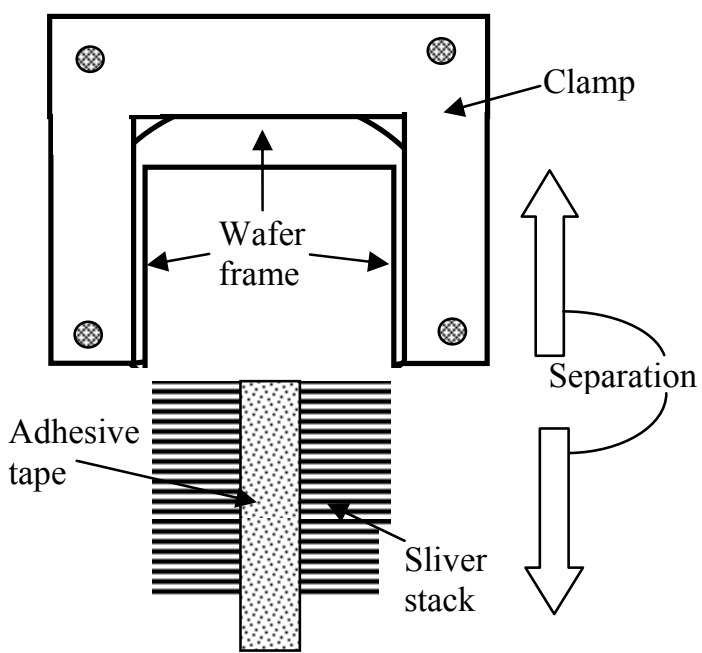

Figure 6. Bulk Sliver cell handling: Separating Sliver cells in a single stack from the wafer frame

The bundle of secured Sliver cells is placed in a carrier, or cassette, of width slightly larger than the length of the Sliver cells. The adhesive tape is then gently removed, in a direction such that the bundle of Sliver cells is caused to conflate to a stack, of height roughly half that of the original stack, simply by the elimination of the space between adjacent cells, as shown in Figure 8. The cassette, containing sufficient Sliver cells to construct a module with a rating of up to $100 \mathrm{~W}$, can be stored until it is needed.

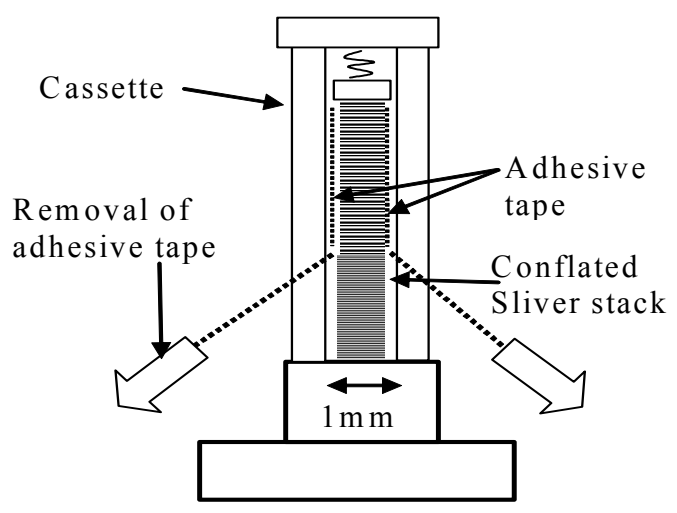

Figure 8. Bulk Sliver cell handling: Transferring Sliver cells and loading a single-stack cassette 
intermittent metal tracks on each crossbeam, and via the intermittent tracks to adjacent cell electrodes, thus forming a functional, series-connected string of 50 Sliver cells in a $100 \times 100 \mathrm{~mm}$ Raft. The process is repeated several thousand times until the Sliver cells in the cassette stacks are exhausted, creating Rafts with a combined rating of several kW.

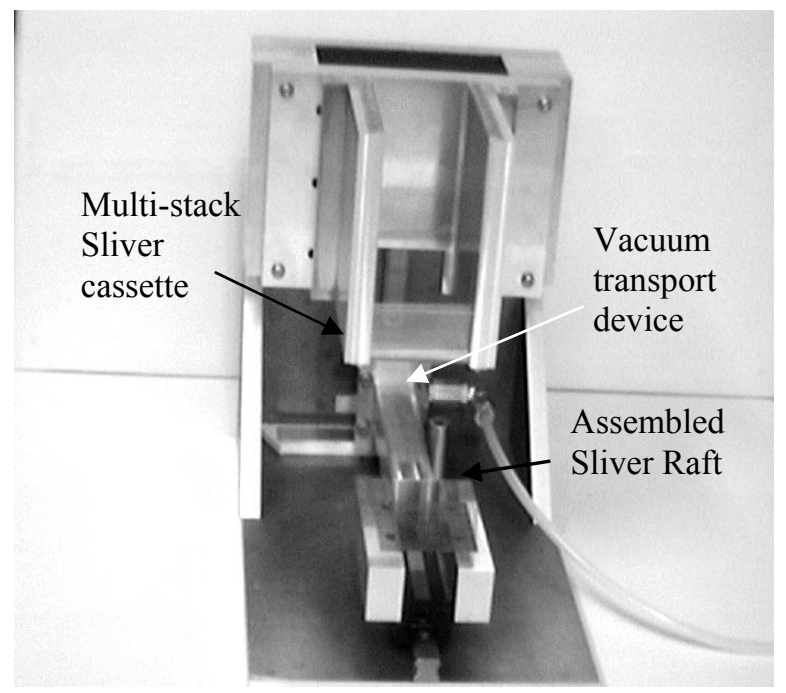

Figure 9. A bench-top prototype of a multi-stack cassette Raft assembly jig.

The modular sub-assembly produces a high voltage - up to $60 \mathrm{~V}$, and a correspondingly low current - as low as $1 / 100^{\text {th }}$ that of a comparable conventional cell, with a total power output better than a conventional cell because of the higher efficiency of Sliver cells ${ }^{18}$.

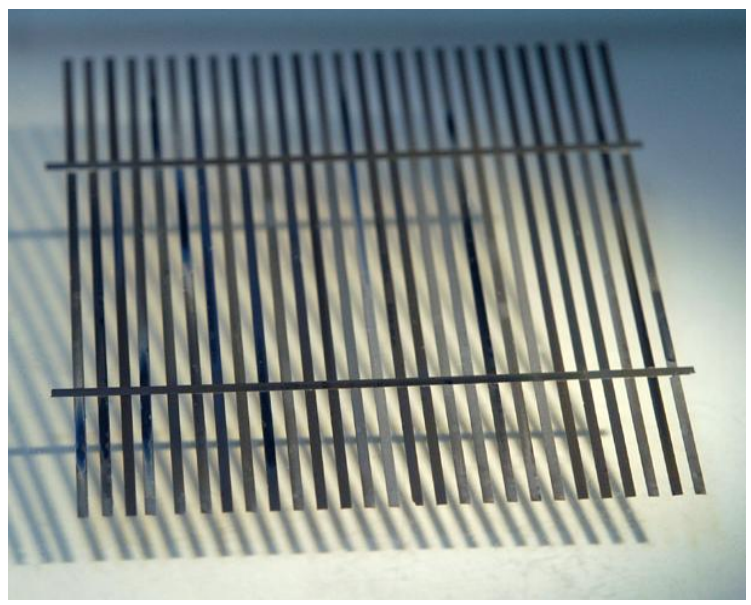

Figure 11. A Raft sub-module, consisting of a group of Sliver cells interconnected by two thin, narrow substrate supports
The use of mono-crystalline silicon as Raft sub-module array support substrates eliminates the problems of differential expansion introduced by the use of dissimilar materials into the Raft structure. Providing the selected supporting medium is very narrow, this structural design also allows the bi-facial properties of Sliver cells to be utilised by placing a scattering reflector on the rear surface of the solar power module to redirect the light that passes through the sub-module arrays back onto the rear surface of the Sliver solar cells.

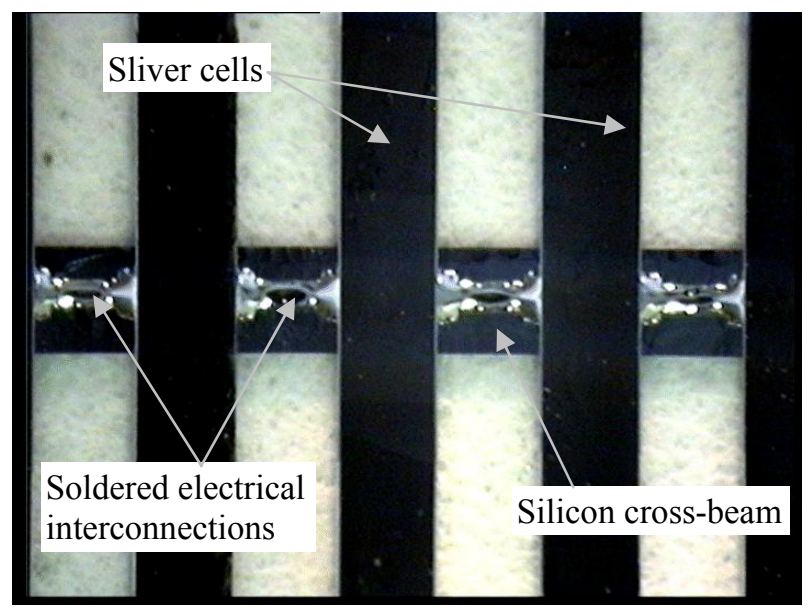

Figure 10. Detailed view of a selective wave-soldered ANU Sliver Raft, formed using a multi-stack cassette.

With implementations of Sliver cell modular sub-assemblies, solder ideally forms the electrical interconnections as well as, in certain implementations, also providing the mechanical structural support for the sub-assembly ${ }^{19}$. The solder can be deposited using a wide variety of methods, including using a wave solder process. A selective wave solder process has the potential to be very rapid because of the low thermal mass of the sub-modules. Figure 10 provides a detailed view of a selective wave soldered Raft where the support structures, or Raft cross-beams, are un-processed silicon Sliver substrates. The Sliver substrates are oxidized to make the surfaces nonconducting. One surface of the substrate is patterned with specially shaped metal pads, that, when soldered, are designed to electrically connect adjacent Sliver cells in series. The design of the pads can be used to control both the quantity and distribution of solder retained on the cross beams during the selective wave solder process ${ }^{19}$. The cross-beams have intermittent metal pads which are used to retain solder during the selective wave soldering process, to form the serial electrical interconnections in the Sliver Raft assembly. 
The sub-module approach offers a means for the low-cost assembly of groups of Sliver cells to form a conventional solar cell analogue. Figure 11 shows a sub-module made from many Sliver cells, both physically and electrically connected using two long, thin, and narrow silicon substrates, referred to as Raft cross-beams. Raft or Sheet sub-modules can be used in place of conventional solar cells to produce a PV module of any desired size, shape, current and voltage, and associated power.

\subsection{Sliver modules}

Modular sub-assemblies can be formed in sizes similar to conventional solar cells, typically $10 \times 10$ to $12 \times 12 \mathrm{~cm}^{2}$. Each sub-module can be incorporated as a conventional solar cell analogue in a photovoltaic module, allowing the use of techniques similar to those used for conventional solar cells and modules to be adapted for testing, binning, handling, assembly, electrical connection, and subsequent encapsulation of Sliver solar cells. The appropriate number of submodules can be deployed to form a photovoltaic module with any desired shape, area, current and voltage characteristics, and associated output power. An example of a Sliver module constructed using Raft sub-modules in shown in Figure 12.

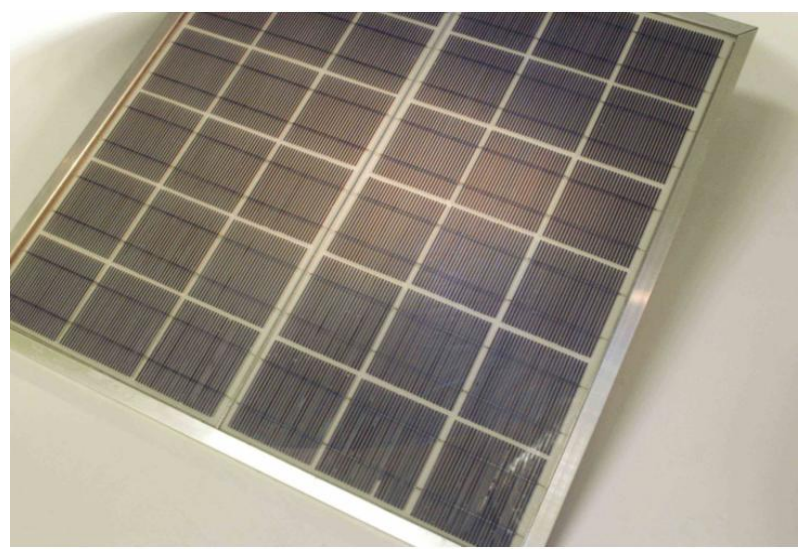

Figure 12. A Sliver module constructed from Raft sub-modules.

A very important advantage of the Raft sub-module approach is that solar power modules constructed using Sliver sub-modules can be manufactured using entirely conventional PV module materials - the Sliver cells, solder and conventional bus-bars, EVA and glass.

The measurement of the efficiency of a large number of individual small Sliver solar cells is both inconvenient and expensive. However, the characteristics of sub-modules can be directly measured, thus effectively allowing dozens to hundreds of small solar cells to be measured together in a single operation and binned according to performance.

Sub-modules will have a large voltage and a correspondingly small current, if the constituent Sliver cells are connected in series. For example, a $12 \times 12 \mathrm{~cm}^{2}$ sub-module composed of sixty $1 \mathrm{~mm}$ wide, series-connected Sliver cells with a gap between each cell of $1 \mathrm{~mm}$ will have a Voc and Jsc of about 40 volts and $70 \mathrm{~mA}$ respectively after encapsulation, and including a Lambertian rear reflector for the module. This compares very favourably with typical figures of $0.63 \mathrm{~V}$ and $5 \mathrm{~A}$ respectively for a conventional cell of similar area.

Sliver Raft and Sheet modules can be constructed to provide high voltage outputs. For example, the module shown in Figure 16 has an output voltage of $45 \mathrm{~V}_{\mathrm{mpp}}$, with twelve $45 \mathrm{~V}$-strings connected in parallel. Alternatively, each string could be connected in series to provide a module output of 540V. Many other intermediate arrangements are also easily implemented.

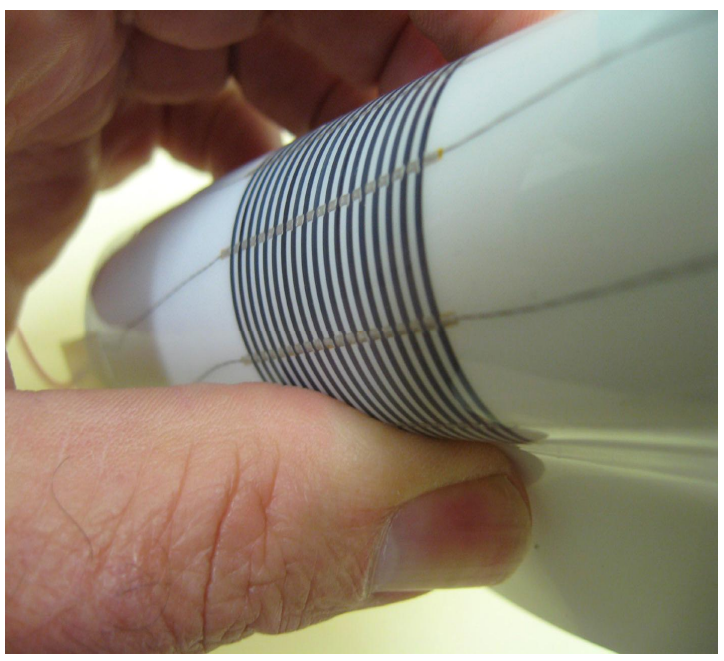

Figure 13. A flexible Sliver module constructed from flexible Raft sub-modules.

Sliver modules have advantages over conventional modules with respect to the elevated temperatures typically experienced during normal operation. Because Sliver cells are high open circuit voltage devices, they have smaller temperature coefficients. Further, through a combination of module design, particularly with Lambertian rear reflector arrangements, and high efficiency Sliver cells, a Sliver module will operate at lower temperatures compared to a conventional module under identical conditions. Hence at operating temperatures well above standard test conditions, Sliver module efficiency will degrade significantly less than an equivalent conventional solar module ${ }^{21}$.

Advantage can be taken of the flexibility of Raft sub-modules fabricated using thin and flexible Sliver solar cells and substrate supports to mount the sub-modules conformally onto a curved supporting structure. The sub-modules can 
optionally be made semi-transparent by spacing the Sliver cells apart. It is difficult to achieve such outcomes using conventional solar cells. The sub-module approach lends itself readily to the fabrication of flexible modules, such as the example in Figure 13.

\section{CONCLUSION}

Novel Sliver cell and unique module design opportunities offer the potential for a 10 to 20 fold reduction in silicon consumption with from 20 to 40 times fewer wafer starts per MW than for conventional wafer-based technologies. Successful commercial implementation of Sliver technology relies upon a robust and high-yield Sliver cell processing sequence and a low-cost, high-throughput method for module construction. These two components go hand-in-hand: the fundamental Sliver properties necessitate the existence of an efficient handling method, while a cost-effective submodule assembly process demands high yield and consistency in finished Sliver cells.

Research at ANU has provided an optimised Sliver cell fabrication process to produce high efficiency cells using a simplified processing sequence that promotes high consistency and a very high yield.

Low-cost equipment and standard PV materials alone can be used to reliably and rapidly produce Sliver sub-module units which can then be easily handled in a similar manner to conventional solar cells.

An optimised Sliver cell processing sequence capable of producing $20 \%+$ cells, when coupled with a robust, low-cost Sliver module construction method, can be expected to significantly reduce the costs of commercial PV modules. The Sliver cell, Raft sub-assembly and Sliver module technologies combined have the potential to provide low-cost solar generated electricity for virtually any application.

Much skilled engineering work is still required to translate the exceptional promise of Sliver technology into commercial reality. However, we believe that the essential building blocks are now in place and that the technology can, if handled appropriately, be successfully transferred to industry.

\section{ACKNOWLEDGMENTS}

Financial support from the Australian Research Council is gratefully acknowledged. The authors would also like to thank members of the Centre for Sustainable Energy Systems for their assistance with the project.

Sliver ${ }^{\circledR}$ is a registered trademark of Origin Energy.

\section{REFERENCES}

[1] M. Rogol, B. Fisher, 'Sun Screen II: Investment opportunities in solar power', Credit Lyonnaise Securities Asia, 2005

[2] S. Reber, A. Eyer, et al., 'Progress in Crystalline Silicon Thin-Film Solar Cell Work at Fraunhofer ISE', 20th European Photovoltaics Solar Energy Conference, Barcelona, (2005).

[3] B. Terheiden, R. Horbelt, and R. Brendel, 'Thin-film Solar Cells and Modules from the Porous Silicon Process Using 6" Si Substrates', 21st European Photovoltaic Solar Energy Conference, Dresden (2006)

[4] A. Aberle, 'Progress in Evaporated Crystalline Silicon Thin-Film Solar Cells on Glass', 4th World Conference on Photovoltaic Energy Conversion, Hawaii, USA, (2006).

[5] K.J. Weber, A.W. Blakers, M.J. Stocks, J. H. Babaei, V.A. Everett, A.J Neuendorf, and P.J. Verlinden, “A Novel Low Cost, High Efficiency Micromachined Silicon Solar Cell”, Electron Device Letters 25, 37, 2004

[6] A.W. Blakers, M.J. Stocks, K.J. Weber, V. Everett, J. Babaei, P. Verlinden, M. Kerr, M. Stuckings and P. Mackey, "Sliver® Solar Cells", 13th NREL workshop on Crystalline Si Materials and Processing, Vail Colorado, 2003

[7] K.J. Weber, A.W. Blakers, V. Everett and E. Franklin, "Results of a cost model for Sliver® cells", 21st EC Photovoltaic Solar Energy Conference, Dresden, September 2006

[8] P. A. Iles and S. I. Soclof, Applied Solar Energy Corporation, City of Industry, Ca.91746, 'Silicon Solar Cells Formed by Orientation Dependent Slicing', Proceedings $15^{\text {th }}$ IEEE Photovoltaic Specialist Conference, p.115-119, 1981.

[9] K.J. Weber and A.W, Blakers, , "Semiconductor Processing", PCT/AU01/01546, 2001.

[10] K.J. Weber, J. MacDonald, V.A. Everett, P.N.K. Deenapanray, M.J. Stocks, and A.W. Blakers, "Modeling of Sliver Modules Incorporating a Lambertian Rear Reflector", $19^{\text {th }}$ European Photovoltaic Solar Energy Conference, Paris, France, 2004. 
[11] K Weber and A Blakers "Semiconductor Texturing Process", European Patent EP1461834.

[12] K. Weber and A. Blakers, “A Novel Silicon Texturisation Method Based on Etching Through a Silicon Nitride Mask", Progress in Photovoltaics, vol. 13: p.691-695, 2005.

[13] Hao Jin and K.J. Weber, "The effect of low pressure chemical vapor deposited silicon nitride on the Si-SiO2 interface of oxidised silicon wafers" Journal of The Electrochemical Society, 154 (1) H5-H8 (2007).

[14] Hao Jin, K.J.Weber, and A.W.Blakers, "Passivation of LPCVD nitride silicon stacks by atomic H", IEEE 4th World Conference on Photovoltaic Energy Conversion, Hawaii, May 2006.

[15] Hao Jin, K.J.Weber, and A.W.Blakers, "Depassivation of Si-SiO2 interface following rapid thermal annealing", IEEE 4th World Conference on Photovoltaic Energy Conversion, Hawaii, May 2006.

[16] Hao Jin, K.J.Weber, and A.W.Blakers, "The effect of a post oxidation in-situ nitrogen anneal on Si surface passivation”, IEEE 4th World Conference on Photovoltaic Energy Conversion, Hawaii, May 2006.

[17] Evan Franklin and Andrew Blakers, "Sliver cells for concentrator systems", $19^{\text {th }}$ European Photovoltaic Solar Energy Conference, Paris, France, 2004.

[18] V. Everett and A.W. Blakers, 2004, "Solar cell (Sliver) sub-module formation", Australian Provisional Patent 2004904478, PCT/AU2005/001193, WO 2006/015430.

[19] V. Everett and A.W. Blakers, 2005, "Solar Cell Interconnection Process", Australian Provisional Patent 2005903172, PCT/AU2006/000840, WO2006133507.

[20] M. Kerr and P. Verlinden, 2005, "Modular sub-assembly of semiconductor strips", WO 2006/079174 A1.

[21] V. Everett, J. Babaei, P. N. K. Deenapanry, K. J. Weber, M. J. Stocks \& A. W. Blakers, The Australian National University, Canberra, Australia; M. J. Kerr, M. F. Stuckings, D. Gordeev, P. J. Verlinden, Origin Energy Solar, Adelaide, Australia, "Characterisation of the Thermal Response of Sliver ${ }^{\circledR}$ Cells and Modules", $19^{\text {th }}$ European Photovoltaic Solar Energy Conference and Exhibition, Paris, France 2004. 